\title{
Inhomogeneous perturbations in the pseudo-Hermitian quantum cosmology
}

\author{
Oleg Novikov ${ }^{1, *}$ \\ ${ }^{1}$ Saint Petersburg State University, 7/9 Universitetskaya nab., St. Petersburg, 199034 Russia
}

\begin{abstract}
We consider the quantum cosmology of the phantom fields within the Wheeler-DeWitt approach using the classically equivalent model with complex potential. The Born-Oppenheimer approximation is used to study the behaviour of the inhomogeneous perturbations over the minisuperspace background. The evolution Hamiltonian is treated as a pseudo-Hermitian one and the conserved inner product is constructed within WKB approximation of the homogeneous wavefunction.
\end{abstract}

\section{Introduction}

While the explanation of the Dark Energy as a cosmological constant remains to be the simplest one it is somewhat puzzling because of the apparent fine-tuning. Thus it may be worthwile to explore the alternative explanations of the Dark Energy as a dynamical matter with equation of state $w=p / \epsilon$ changing during the cosmological evolution. While the present observations [1] put rather strong constraints on the models with $w>-1$ the possibility remains that the Dark Energy is produced by so-called phantom matter with $w<-1$. E.g. one may consider a two-field model with one of the scalar field $\xi$ having wrong-sign kinetic term[2],

$$
L=\frac{1}{2} \partial_{\mu} \phi \partial^{\mu} \phi-\frac{1}{2} \partial_{\mu} \xi \partial^{\mu} \xi-V(\phi, \xi)
$$

The obvious issue with such fields is that the energy of the $\xi$ field is not bounded from below. As result the models are plagued by severe instabilities.

However one may hope that such exotic models may still make sense with non-standard quantization. E.g. some of the seemingly unstable and even complex classical Hamiltonians can still possess purely real positive spectrum if they are symmetric under the following $\mathcal{P} \mathcal{T}$ transformation [3],

$$
\mathcal{P T}: \quad x \mapsto-x, \quad p \mapsto-p, \quad i \mapsto-i
$$

Moreso they generate an unitary evolution for certain non-standard norm,

$$
\left(\psi_{1}, \psi_{2}\right)=\left\langle\psi_{1}|\mathcal{C P} \mathcal{T}| \psi_{2}\right\rangle
$$

Where the new operator $C$ is introduced to make this norm positively-definite. These Hamiltonians actually form a particular subset of the more general so-called pseudo-Hermitian operators related to the Hermitian ones by non-unitary similarity operator $\eta$,

$$
\hat{H}=\eta^{-1} \hat{h} \eta, \quad \hat{h}=\hat{h}^{\dagger}, \quad(\psi, \tilde{\psi})=\left\langle\psi\left|\eta^{\dagger} \eta\right| \tilde{\psi}\right\rangle
$$

\footnotetext{
*e-mail: o.novikov@ spbu.ru
} 
However the Hermitian form usually happens to be very complicated and it is much easier to study such models in the non-Hermitian form.

To apply this idea to the cosmology one may replace (1) with the classically equivalent model to apply this idea to the cosmology by replacing (1) with a classically equivalent model with .

$$
L=\frac{1}{2} \partial_{\mu} \phi \partial^{\mu} \phi+\frac{1}{2} \partial_{\mu} \tilde{\phi} \partial^{\mu} \tilde{\phi}-V(\phi, i \tilde{\phi}),
$$

Where the new field $\tilde{\phi}=-i \xi$ has positive kinetic energy but complex potential. The classical solution that has real energy and pressure will be purely imaginary and thus will coincide with the usual phantom solution. In contrast it is conjectured that the perturbations should be considered along the real axis, $\tilde{\phi}=i \xi_{\text {class }}+\delta \phi$. It may be shown that such perturbations near classical trajectory happen to possess positively definite effective Hamiltonian $[4,5]$. To separate such fields from the usual phantoms we coin a new name-PTom. A realization of this idea as a fully quantum model restricted to the homogeneous minisuperspace was considered in [6] and the similarity operator for the inhomogeneous perturbations was constructed in [7]. In this paper we propose a generalization of the ordinary WKB-current to construct a conserved inner product that may be used then to compute the correlation functions.

\section{Evolution of the inhomogeneous fluctuations}

Let us consider the following model of two scalar fields: quintessence and PTom,[6]

$$
\begin{aligned}
S & =\int d^{4} x \sqrt{-g}\left(-\frac{1}{2 \kappa^{2}} R+\frac{1}{2} M_{\Phi \Phi} \partial_{\mu} \Phi \partial^{\mu} \Phi+\frac{1}{2} M_{\tilde{\Phi} \tilde{\Phi}} \partial_{\mu} \tilde{\Phi} \partial^{\mu} \tilde{\Phi}\right. \\
& \left.+i M_{\Phi \tilde{\Phi}} \partial_{\mu} \Phi \partial^{\mu} \tilde{\Phi}-V(\Phi, \tilde{\Phi})\right)
\end{aligned}
$$

where $\kappa^{2}=8 \pi G=M_{P l}^{-2}$. We take all parameters to be real and assume that $(V(\Phi,-\tilde{\Phi}))^{*}=$ $V(\Phi, \tilde{\Phi})$ to preserve the following symmetry,

$$
\mathcal{P T}: \quad t \mapsto-t, \quad i \mapsto-i, \quad \Phi \mapsto \Phi, \quad \tilde{\Phi} \mapsto-\tilde{\Phi} .
$$

Note that in the Hamiltonian formulation and after the quantization $t \mapsto-t$ means actually the following transformation of the momenta,

$$
\mathcal{P T}: \quad p_{g_{\mu \nu}} \mapsto-p_{g_{\mu \nu}}, \quad p_{\Phi} \mapsto-p_{\Phi}, \quad p_{\tilde{\Phi}}=p_{\tilde{\Phi}}
$$

The transverse ( $3 \mathrm{~d}$ tensor and vector) modes decouple from the longitudinal ( $3 \mathrm{~d}$ scalar) ones at the quadratic level. We will restrict ourselves only to the longitudinal inhomogeneous sector as the pseudo-Hermitian construction is not expected to appear in the tensor and vector modes before taking into account non-linearities. We consider the following metric and field ansatz,

$$
\begin{gathered}
d s^{2}=\left(N^{2}(t)+s(t, x)\right) d t^{2}+2\left(\partial_{k} v(t, x)\right) d t d x^{k}-e^{2 \rho}\left(\delta_{i j}+h(t, x) \delta_{i j}+\partial_{i} \partial_{j} E(t, x)\right) d x^{i} d x^{j} \\
\Phi(t, \vec{x})=\Phi(t)+\phi(t, \vec{x}), \quad \tilde{\Phi}=\tilde{\Phi}(t)+\tilde{\phi}(t, \vec{x})
\end{gathered}
$$

The variables $\left\{Q_{A}\right\} \equiv\{N, \rho, \Phi, \tilde{\Phi}\}$ are the coordinates on the restricted minisuperspace whereas $\left\{\chi_{a}\right\} \equiv\{s, v, h, E, \phi, \tilde{\phi}\}$ are treated as small fluctuations. We will take the following partial gauge fix,

$$
h=E=0
$$


To proceed we perform IR regulazation to the finite volume $\mathcal{V}$. Let us decompose the remaing fluctuation variables into the superposition of the orthonormal eigenfunctions of the Laplace operator acting on this regularized space.

$$
\chi_{a}(t, \vec{x})=\sum_{n} \chi_{a}^{(n)}(t) f_{n}(\vec{x}), \quad-\Delta f_{n}(\vec{x})=\Omega_{n}^{2} f_{n}(\vec{x}), \quad \int_{\mathcal{V}} d^{3} x f_{n}(\vec{x}) f_{m}(\vec{x})=\delta_{n m} .
$$

The variables $N, v$ and $s$ are not dynamical and generate constraints. As usual in general relativity one of the constraints is the Hamiltonian that up to the quadratic order in fluctuations takes the form,

$$
H=\mathcal{V} H_{0}+\sum_{n} H_{2}^{(n)} \simeq 0
$$

The Dirac quantization of this constraint leads to the Wheeler-DeWitt (WdW) equation in the following form,

$$
\left(\mathcal{V} \hat{H}_{0}+\sum_{n} \hat{H}_{2}^{(n)}\right) \Psi\left(\left\{Q_{A}\right\},\left\{\chi_{a}\right\}\right)=0,
$$

Then we use the Born-Oppenheimer approximation [8]-[15] and separation of the fluctuation variables corresponding to the different inhomogeneous modes,

$$
\Psi\left(\left\{Q_{A}\right\},\left\{\chi_{a}\right\}\right) \simeq \Psi_{0}\left(\left\{Q_{A}\right\}\right) \prod_{n} \Psi_{2}^{(n)}\left(\left\{Q_{A}\right\},\left\{\chi_{a}^{(n)}\right\}\right)
$$

Decomposing the WdW equations into different orders of $\kappa^{3} / \mathcal{V}$ we first obtain the WdW equation in the restricted minisuperspace,

$$
\hat{H}_{0} \Psi_{0}=\left(\frac{\kappa^{2}}{12 \mathcal{V}^{2}} \partial_{\rho}^{2}-\frac{M_{\tilde{\Phi} \tilde{\Phi}}}{2 \mathcal{V}^{2} \mathcal{D}} \partial_{\Phi}^{2}-\frac{M_{\Phi \Phi}}{2 \mathcal{V}^{2} \mathcal{D}} \partial_{\tilde{\Phi}}^{2}+i \frac{M_{\Phi \tilde{\Phi}}}{\mathcal{V}^{2} \mathcal{D}} \partial_{\Phi} \partial_{\tilde{\Phi}}+V(\Phi, \tilde{\Phi})\right) \Psi_{0}=0
$$

The large volume limit $\mathcal{V} / \kappa^{3} \rightarrow \infty$ works as a quasiclassical limit. Assuming that we consider the quasiclassical wavepacket far from the turning point we may use the WKB approximation for the minisuperspace part of the wavefunction,

$$
\Psi_{0}\left(\left\{Q_{A}\right\}\right)=\mathcal{A}\left(\left\{Q_{A}\right\}\right) \exp \left(i \mathcal{V} S\left(\left\{Q_{A}\right\}\right)\right)
$$

We will neglect backreaction assuming that $\hat{H}_{0} \Psi_{2}^{(n)}=0$. Then for the fluctuation part of the wavefunction we obtain the Schrödinger equation,

$$
\frac{i}{\mathcal{V}} \partial_{\tau} \Psi_{2}^{(n)}=\hat{H}_{2}^{(n)} \Psi_{2}^{(n)}
$$

where $\partial_{\tau}$ is the derivative in the so-called WKB-time that is directed along the classical trajectory[8],

$$
\frac{1}{\mathcal{V}} \partial_{\tau}=G^{A B}\left(\partial_{A} S\right) \partial_{B}
$$

with $\mathcal{G}^{A B}$ being the metric on the minisuperspace $\left\{Q_{A}\right\}$,

$$
\mathcal{G}^{A B} X_{A} Y_{B} \equiv \frac{\kappa^{2}}{12} X_{\rho} Y_{\rho}-\frac{M_{\tilde{\Phi} \tilde{\Phi}}}{2 \mathcal{D}} X_{\Phi} Y_{\Phi}-\frac{M_{\Phi \Phi}}{2 \mathcal{D}} X_{\tilde{\Phi}} Y_{\tilde{\Phi}}+i \frac{M_{\Phi \tilde{\Phi}}}{2 \mathcal{D}}\left(X_{\Phi} Y_{\tilde{\Phi}}+X_{\tilde{\Phi}} Y_{\Phi}\right)
$$

Looking at this equation we may interpret $H_{2}^{(n)}$ as the effective Hamiltonian for the fluctuation part evolution along the family of the classical solutions defined by WKB-action $S$. 


\section{The pseudo-Hermitian construction}

In our model $H_{2}^{(n)}$ is real only when computed on the purely imaginary trajectories. For the complex trajectories we should treat it as the time-dependent pseudo-Hermitian Hamiltonian using the generalization of (4) following [16],

$$
\hat{H}_{2}^{(n)}=\left(\eta_{n}\left(\left\{Q_{A}\right\}\right)\right)^{-1} \hat{h}^{(n)}\left(\left\{Q_{A}\right\}\right) \eta_{n}\left(\left\{Q_{A}\right\}\right)-i\left(\eta_{n}\left(\left\{Q_{A}\right\}\right)\right)^{-1} \partial_{\tau}\left(\eta_{n}\left(\left\{Q_{A}\right\}\right)\right),
$$

where both $\hat{h}^{(n)}$ and $\eta_{n}$ are Hermitian operators. In the time-dependent case the resulting non-Hermitian Hamiltonian ceases to be observable even under the norm $\left\langle\psi\left|\eta^{\dagger} \eta\right| \phi\right\rangle$ however generates unitary evolution.

Let us now restrict our consideration to the specific potential and kinetic matrix that allows separation of the homogeneous variables $[6,17]$,

$$
V(\Phi, \tilde{\Phi})=V e^{\Phi}-\tilde{V} e^{i \tilde{\Phi}}, \frac{M_{\Phi \tilde{\Phi}}}{\mathcal{D}}=6 \kappa^{2}, D=\frac{M_{\tilde{\Phi} \tilde{\Phi}}}{\mathcal{D}}-6 \kappa^{2}, \tilde{D}=\frac{M_{\Phi \Phi}}{\mathcal{D}}+6 \kappa^{2}
$$

In the weak gravity limit $\kappa \rightarrow 0$ after solving the constraint $\mathcal{H}_{s}^{(n)}$ the effective Hamiltonian greatly simplifies with $\phi$ and $\tilde{\phi}$ being decoupled,

$$
\begin{gathered}
\hat{H}_{2}^{(n)}=\hat{H}_{\phi, 2}^{(n)}+\hat{H}_{\tilde{\phi}, 2}^{(n)}+O(\kappa), \\
\hat{H}_{\phi, 2}^{(n)}=\frac{D}{2}\left(p_{\phi}^{(n)}\right)^{2}+\left[\Omega^{2}+\frac{V}{2} e^{6 \rho+\Phi}\right]\left(\phi^{(n)}\right)^{2}, \quad \hat{H}_{\tilde{\phi}, 2}^{(n)}=\frac{\tilde{D}}{2}\left(\tilde{p}_{\phi}^{(n)}\right)^{2}+\left[\Omega^{2}+\frac{\tilde{V}}{2} e^{6 \rho+i \tilde{\Phi}}\right]\left(\tilde{\phi}^{(n)}\right)^{2}
\end{gathered}
$$

Because $\Phi$ and $\phi$ are ordinary Hermitian fields $\hat{H}_{\phi, 2}^{(n)}$ is manifestly Hermitian and $\eta_{n}$ may be be nontrivial only on the $\tilde{\phi}$ sector. The remaining part $\hat{H}_{\tilde{\phi}, 2}$ is a non-Hermitian time-dependent Swanson Hamiltonian[18, 19]. For the purely imaginary solution $\tilde{\Phi}=i \xi_{\text {class }}$ this is simply a Hermitian oscillator with time-dependent frequency. Hence the simplest option is to relate the complex trajectory to some purely imaginary trajectory $\tilde{\Phi} \simeq i \xi_{\text {class }}+\delta \tilde{\Phi}$,

$$
\hat{h}_{\tilde{\phi}}^{(n)}=\left.\hat{H}_{2, \tilde{\phi}}^{(n)}\right|_{\tilde{\Phi}=i \xi_{0}}, \eta_{n} \equiv e^{X_{n}}=\exp \left[\alpha_{n}(\delta \tilde{\Phi}) p_{\tilde{\phi}}^{2}+\beta_{n}(\delta \tilde{\Phi}) \tilde{\phi}^{2}+\gamma_{n}(\delta \tilde{\Phi})\left(\tilde{p}_{\phi} \tilde{\phi}+\tilde{\phi} \tilde{p}_{\phi}\right)\right]
$$

The choice of the contour that determines $i \xi_{\text {class }}$ with which the complex solution is related defines our quantum model. In perturbation theory we may treat $\delta \tilde{\Phi}$ as small purely real parameter and find $\eta$ explicitly [7].

\section{The conserved inner product}

Let us start with the ordinary Wheeler-DeWitt quantum cosmology. The construction of the inner product is an open problem intricately related to the problem of time. The simplest conserved current, namely Klein-Gordon current,

$$
J_{A}=\Psi_{2}^{*} \partial_{A} \Psi_{1}-\Psi_{1}\left(\partial_{A} \Psi_{2}\right)^{*},
$$

is not positively definite and thus can't be used to define probabilities on the generic states. However if we restric ourselves to the states where one can factor out the homogeneous part as a WKB wavepacket [8],

$$
\Psi\left(\left\{Q_{A}\right\},\left\{\chi_{a}\right\}\right) \simeq \mathcal{A}\left(Q_{A}\right) e^{i \mathcal{V} S\left(Q_{A}\right)-\mathcal{V} \mathcal{R}\left(Q_{A}\right)} \psi_{2}\left(\left\{Q_{A}\right\},\left\{\chi_{a}\right\}\right),
$$


where $\partial_{A} S \gg \partial_{A} \mathcal{R}$ i.e. we consider the region near the center of the wavepacket. Let us now substitute two states of this form with different $\mathcal{R}$ and $\mathcal{A}$ but same $S$ into (26) and integrate $J_{\tau}$ over the surface $\Sigma_{0}$ of constant $S$ in the minisuperspace $\left\{Q_{A}\right\}$,

$$
\left\langle\Psi_{1} \mid \Psi_{2}\right\rangle_{\Sigma_{0}}=\int_{\Sigma_{0}} d \Sigma\left[\mathcal{A}_{1}\left(\left\{Q_{A}\right\}\right)\right]^{*} \mathcal{A}\left(\left\{Q_{A}\right\}\right)\left\langle\psi_{2}^{(1)} \mid \psi_{2}^{(2)}\right\rangle_{\left\{Q_{A}\right\}}
$$

where

$$
\left\langle\psi_{2}^{(1)} \mid \psi_{2}^{(2)}\right\rangle_{\left\{Q_{A}\right\}}=\left(\prod_{n} \int_{-\infty}^{+\infty} d \chi_{a}^{(n)}\right)\left[\psi_{2}^{(1)}\left(\left\{Q_{A}\right\},\left\{\chi_{a}\right\}\right)\right]^{*} \psi_{2}^{(2)}\left(\left\{Q_{A}\right\},\left\{\chi_{a}\right\}\right) .
$$

Consider now the family of surfaces $\Sigma_{\tau}$ that are obtained by the classical evolution of the initial surface $\Sigma_{0}$ along the family of classical solutions corresponding to the function $S$. Then one can show that this inner product is conserved as long as $\partial_{A} S \gg \partial_{A} \mathcal{R}$ i.e. $\Sigma_{\tau}$ stays sufficiently close to the center of the wavepacket. This may be used to define conditional probabilities.

If we restrict ourselves to the states in the form (27) the inner product may be generalized in a pseudo-Hermitian fashion. First we note that the similarity operator for the fluctuation part obtained above defines the modified norm conserved along the classical trajectory,

$$
\left(\psi_{2}^{(1)} \mid \psi_{2}^{(2)}\right)_{\left\{Q_{A}\right\}}=\left\langle\psi_{2}^{(1)}\left|\eta\left(Q_{A}\right)^{\dagger} \eta\left(Q_{A}\right)\right| \psi_{2}^{(2)}\right\rangle_{\left\{Q_{A}\right\}}
$$

To deal with the minisuperspace part we assume that the action $S$ is symmetric under $\mathcal{P} \mathcal{T}$ symmetry. This means that the WKB-time $\tau$ can also be defined in $\mathcal{P} \mathcal{T}$-symmetric fashion. From $\mathcal{P} \mathcal{T}$-symmetry of $\tau$ and $\hat{H}_{2}^{(n)}$ follows that the similarity operator $\eta$ is also symmetric under the If the integration surface $\Sigma_{0}$ is also chosen to be $\mathcal{P} \mathcal{T}$-symmetric we can define the conserved inner product as,

$$
\left(\Psi_{1} \mid \Psi_{2}\right)_{\Sigma_{0}}=\int_{\Sigma_{0}} d \Sigma\left[C \mathcal{P} \mathcal{T} \mathcal{A}_{1}\left(\left\{Q_{A}\right\}\right)\right]^{*} \mathcal{A}\left(\left\{Q_{A}\right\}\right)\left(\psi_{2}^{(1)} \mid \psi_{2}^{(2)}\right)_{\left\{Q_{A}\right\}}
$$

As usual the new operator $C$ has to be introduced so that the proposed inner product is not positively definite. It should commute with $\mathcal{P} \mathcal{T}$ and be conserved i.e. $\partial_{\tau} C=0$. The standard approach would be to consider a complete set of solutions of the homogeneous Wheeler-DeWitt equation,

$$
\Psi_{k}=\mathcal{A}_{k} e^{i \mathcal{V} S_{k}-\mathcal{V} \mathcal{R}_{k}}, \quad \mathcal{P} \mathcal{T} \mathcal{A}_{k}= \pm \mathcal{A}_{k}, \quad\left(\mathcal{A}_{k}, \mathcal{A}_{l}\right)= \pm \delta_{l k}
$$

where for each solution $\partial_{A} S_{k} \gg \partial_{A} R_{k}$ and introduce the operator as,

$$
C \mathcal{A}=\sum_{k} \mathcal{A}_{k}\left(\mathcal{A}_{k}, \mathcal{A}\right)
$$

However it is unlikely that such set exists for sufficiently generic wavepackets not violating our approximations. Thus to use the proposed inner product one is restricted to the rather special class of the wavefunctions e.g. only $\mathcal{P T}$-symmetric ones for which one may take $C=1$.

\section{Acknowledgments}

The work is done with financial support by grant RFBR, project 16-02-00348, grant RFBR, project 18-02-00264 and also travel grant of Saint Petersburg State University. The author is grateful to A.A.Andrianov, Chen Lan and Yi-Fan Wang for helpful discussions. 


\section{References}

[1] P. A. R. Ade et al. [Planck Collaboration], Astron. Astrophys. 594 A14 (2016)

[2] Cai Y.F., Saridakis E.N., Setare M.R. and Xia J.Q., Phys. Rept. 493 p. 1 (2010)

[3] Bender C.M., Rept. Prog. Phys. 70 p. 947 (2007)

[4] Andrianov A.A., Cannata F. and Kamenshchik A.Y., Int. J. Mod. Phys. D 15 p. 1299 (2006)

[5] Andrianov A.A., Cannata F. and Kamenshchik A.Y., J. Phys. A. 39 p. 9975 (2006)

[6] Andrianov A.A., Lan C., Novikov O.O., In: Bagarello F., Passante R., Trapani C. (eds) Non-Hermitian Hamiltonians in Quantum Physics. Springer Proceedings in Physics. V. 184. (Springer, Cham. 2016) p.29

[7] O. O. Novikov, Phys. Part. Nucl. Lett. 15 p. 353 (2018)

[8] Kiefer C., Quantum gravity (Oxford Uni. Press, 2007) p. 168.

[9] Brout R., Found. Phys. 17 p. 603 (1987)

[10] Brout R. and Venturi G., Phys. Rev. D 39 p. 2436 (1989)

[11] Kamenshchik A.Y., Tronconi A. and Venturi G., Phys. Lett. B. 726 p. 518 (2013)

[12] Kamenshchik A.Y., Tronconi A. and Venturi G., Phys. Lett. B. 734 p. 72 (2014)

[13] Kiefer C. and Kraemer M., Phys. Rev. Lett. 108 p. 021301 (2012)

[14] Brizuela D., Kiefer C. and Kraemer M., Phys. Rev. D. 94 P. 123527 (2016)

[15] Kamenshchik A.Y., Tronconi A. and Venturi G., Class.Quant.Grav. 35 p. 015012 (2018)

[16] Fring A. and Moussa M.H.Y., Phys. Rev. A. 93 p. 042114 (2016)

[17] Andrianov A.A., Novikov O.O., Lan C., Theor. Math. Phys. 184 p. 1224 (2015)

[18] Fring A. and Moussa M.H.Y., Phys. Rev. A. 94 p. 042128 (2016)

[19] Maamache M., Djeghiour O.K., Mana N., Koussa W., arXiv: 1705.06341 